\title{
Long Noncoding RNA SOX2-OT Aggravates Doxorubicin-Induced Apoptosis of Cardiomyocyte by Targeting miR-942-5p/DP5
}

This article was published in the following Dove Press journal: Drug Design, Development and Therapy

\author{
Haining Wang' \\ Xiule Lin' \\ Jilin $\mathrm{Li}^{2}$ \\ Guoning Zeng' \\ Tan $X u^{l}$ \\ 'Department of Cardiovascular Medicine, \\ The First Affiliated Hospital of Shantou \\ University Medical College, Cardiac Care \\ Unit (CCU), Shantou, Guangdong \\ Province, 5I504I, People's Republic of \\ China; ${ }^{2}$ Department of Cardiovascular \\ Medicine, The Second Affiliated Hospital \\ of Shantou University Medical College, \\ Shantou, Guangdong Province, 5I5000, \\ People's Republic of China
}

Background: Long non-coding RNAs (LncRNAs) play important roles in doxorubicin (DOX)-induced apoptosis of cardiomyocytes. However, the function of lncRNA SOX2-OT is unclear. This study was carried out to investigate the function of SOX2-OT in doxorubicininduced cardiomyocyte apoptosis.

Methods: qRT-PCR and immunoblotting were used to detect the expression levels of SOX2-OT, miR-942-5p and death protein-5 (DP5) in DOX-treated primary cardiomyocytes and rat models. The relationship among miR-942-5p, SOX2-OT, and DP5 was explored by luciferase reporter assay. The effects of SOX2-OT, miR-942-5p and DP5 on doxorubicininduced cardiomyocyte apoptosis were evaluated by Annexin V-FITC/PI method and caspase-3 activity assay. The effect of SOX2-OT on cardiomyocyte apoptosis was analyzed by TUNEL staining and echocardiography.

Results: SOX2-OT and DP5 were highly expressed, while miR-942-5p was down-regulated in DOX-treated primary cardiomyocytes and rat model. SOX2-OT can upregulate DP5 as a sponge of miR-942-5p, which was a direct target of miR-942-5p. In addition, miR-942-5p reversed the protective effect of knockdown of SOX2-OT on cardiomyocytes by inhibiting the expression of DP5 in vitro and in vivo.

Conclusion: Knockdown of SOX2-OT down-regulated DP5 via sponging miR-942-5p and inhibiting DOX-induced apoptosis of primary cardiomyocytes.

Keywords: IncRNA SOX2-OT, miR-942-5p, DP5, cardiomyocyte, apoptosis

\section{Introduction}

Doxorubicin (DOX) is an anthracycline anticancer antibiotic. ${ }^{1,2}$ It is widely used as a chemotherapeutic agent in the treatment of various human tumors including breast cancer, lymphoma, lung cancer and leukemia. ${ }^{3,4}$ However, the clinical use of DOX is highly limited by cumulative and irreversible cardiomyopathy that occurs following DOX treatment. The pathogenesis of DOX-induced cardiac muscle dysfunction is complex. ${ }^{5}$ Previous studies have found that cardiomyocyte apoptosis has some common features with cardiomyocyte differentiation, and low concentration of DOX can induce stem cell to differentiate into cardiomyocytes, while high concentration of DOX can induce cardiomyocyte apoptosis. ${ }^{6-8}$ To date, there is no effective treatment for DOX-induced cardiotoxicity and heart failure. ${ }^{9}$ Understanding the mechanism of DOX-induced cardiomyocyte death will provide insights into the reduction of reducing cardiotoxicity and protection of heart function.

\footnotetext{
Correspondence: Haining Wang Department of Cardiovascular Medicine, The First Affiliated Hospital of Shantou University Medical College, CCU, Shantou City, Guangdong Province, 5 I504I, People's Republic of China Tel +86-(0754)88258290 Email wgl424@I63.com

Jilin Li

Department of Cardiovascular Medicine, The Second Affiliated Hospital of Shantou University Medical College, Shantou, Guangdong Province, 515000, People's Republic of China

Email xnjgbezpruec18@I63.com
} 
Long non-coding RNAs (LncRNAs) are greater than 200 nucleotides in length. ${ }^{10}$ Studies have reported altered expression of lncRNAs under conditions of cardiac development and function maintenance as well as normal physiological conditions of the vascular system. ${ }^{11}$ An increasing number of evidence has shown that IncRNAs play critical roles in coronary heart disease, congenital heart disease, myocardial disease, cardiac hypertrophy, myocardial fibrosis and heart failure. ${ }^{12,13}$ LncRNA SOX2OT is located on human chromosome 3q26.3 (chr3q6.3) and is essential for maintaining the pluripotency of selfrenewing and undifferentiated embryonic stem cells. ${ }^{14}$ Studies have shown that SOX2-OT is closely related with the occurrence of various tumors. ${ }^{15}$ For example, high expression levels of SOX2-OT are associated with malignancy and poor prognosis. ${ }^{16}$ However, the biological function of SOX2OT in doxorubicin-induced apoptosis of cardiomyocytes is unclear.

MicroRNAs (miRNAs) are a class of endogenous noncoding small RNAs. ${ }^{17,18}$ Extensive studies have shown that miRNAs are closely related to cardiovascular physiology and pathology. ${ }^{19,20}$ Non-coding RNAs including miRNAs involve pathways such as myocardial apoptosis, myocardial hypertrophy, myocardial fibrosis, and inhibition of neovascularization after acute myocardial infarction to accelerate pathological processes. ${ }^{21,22}$ MiR-942-5p was reported to be downregulated in a variety of diseases, however, its function in the doxorubicin-induced cardiomyocyte apoptosis and the regulatory mechanisms are not clear. ${ }^{23}$ The expression of death protein 5 (DP5) is inhibited or downregulated in tissues, which promotes the invasion and spread of cells. ${ }^{24}$ It has been shown that DP5 is closely related to cell transformation, inflammation, apoptosis, proliferation and autophagy. ${ }^{25}$ Therefore, it was speculated that SOX2OT may regulate the progression of doxorubicin-induced cardiomyocyte apoptosis through the miR-942-5p/DP5 axis. The main goal of this study was to investigate the regulation of SOX2OT in doxorubicininduced cardiomyocyte apoptosis, which provides a theoretical basis for finding new drug targets.

\section{Materials and Methods}

\section{Cell Isolation and Culture}

Primary cultured cardiomyocytes (PC) were prepared as described in the literature. ${ }^{26}$ The SD rats of $1-3 \mathrm{~d}$ were taken out and hearts were aseptically removed. Cells were digested with $1 \mathrm{~g} / \mathrm{L}$ of sterile collagenase II to prepare cell suspension.
Cells were suspended in Dulbecco's Modified Eagle Medium/ F-12 (GIBCO, USA) containing 10\% FBS, $100 \mathrm{U} / \mathrm{mL}$ penicillin, and $100 \mathrm{mg} / \mathrm{mL}$ streptomycin. Cells were then incubated in $95 \%$ air and $5 \% \mathrm{CO}_{2}$ at $37^{\circ} \mathrm{C}$ for 24 or $48 \mathrm{~h}$, and the adherent cells were washed twice in phosphate-buffered saline (PBS). The cultures were depleted of erythroid progenitor cells through the removal of cells that did not adhere to the culture dish at medium change. Cells were harvested at $80 \%$ confluence with $0.25 \%$ trypsin and passaged at a ratio of $1: 3$. The medium was changed twice a week. The primary cardiomyocytes were harvested after cultured for 21-28 d. This study was approved by the Animal Care and Use Committee of the First Affiliated Hospital of Shantou University Medical College. All experimental procedures were performed following the guidelines for the use of laboratory animals in China.

\section{Cell Transfection}

PcDNA3.1-SOX2-OT (SOX2-OT; catalog: \#39453), siRNA for SOX2-OT (si-SOX2-OT; 5'-GGAGAUUGUGACC UGGCUU-3'), miR-942-5p mimic (catalog: \#4263066), miR-942-5p agomir (catalog: \#245813), siRNA against DP5 (si-DP5; 5'-GATGTGAACTCTGAGACTTTGTGAT-3'), miRNA negative control (miR-NC; 5'-GGUAUUCCUC UCGUUUACA-3') or negative control were obtained from Shanghai GenePharma Co., Ltd. (Shanghai, China). For cell transfection, primary cardiomyocytes were plated in 24-well plates and transfected with $100 \mathrm{ng}$ target vector using Lipofectamine 2000 (Invitrogen). The medium was replaced with fresh medium after $6 \mathrm{~h}$. Subsequent experiments were performed at $24 \mathrm{~h}$ after transfection.

\section{In vivo Studies}

Male Wister rats (10-11 weeks old) were purchased from Shantou University Medical College Animal Research Center. This study was performed in strict accordance with the NIH guidelines for the care and use of laboratory animals (NIH Publication No. 85-23 Rev. 1985). All animal procedures were performed in accordance with the Guidelines for Care and Use of Laboratory Animals of "the First Affiliated Hospital of Shantou University Medical College" and approved by the Animal Ethics Committee of "Animal Ethical and Welfare Committee (AEWC)".

To obtain an optimal efficacy of cardiac knockdown of SOX2-OT and overexpression of miR-942-5p, the rAAV9 (Biowit Technologies Co., Ltd, China; catalog: \#7863)mediated gene delivery was achieved through intramyocardial injection. Twenty microliters containing $5 \times 10^{12}$ 
GC (genome copies) of rAAV9 vectors were injected in five randomly chosen sites within left ventricles. After injection for 1 week, the rats were injected intraperitoneally with $2.5 \mathrm{mg} / \mathrm{kg}$ DOX, and a cardiomyopathy model was induced in six equal doses over a period of 2 weeks. Two weeks after the last injection of DOX, cardiac function was evaluated by echocardiography and hemodynamics, and myocardial specimens were subjected to molecular biological analyses. Rats in the control group (Control, $\mathrm{n}=10$ ) were intraperitoneally injected with saline solution. Four weeks after the first injection of DOX, animals were anesthetized with pentobarbital $(40 \mathrm{mg} / \mathrm{kg})$ intraperitoneally and echocardiographic parameters were analyzed using a MicroUltrasound system (Vevo 770, VisualSonics Inc.). Linear sensors were used to analyze LVFS and LVEF. The rats were euthanized through intraperitoneal administration of a $120 \mathrm{mg} / \mathrm{kg}$ pentobarbital solution. Rat death was verified with no heartbeat, no spontaneous breathing for $2 \mathrm{~min}$ and no blink reflex. Then, the hearts were collected for qRTPCR, Western blotting, and terminal deoxynucleotidyl transferase-mediated dUTP nick end labeling (TUNEL).

\section{CCK-8 Assay}

Cell counting Kit-8 kit (CCK-8, MyBioSource) was used to detect the viability of primary cardiomyocytes. In brief, $10^{4}$ cells were seeded into a 96 -well plate at $37^{\circ} \mathrm{C}$. At 0 , $12,24,48$, and $72 \mathrm{~h}$ after experimental treatment, the cells were incubated with $100 \mu \mathrm{L}$ CCK-8 solution for 2 h. Finally, the OD values at $450 \mathrm{~nm}$ were recorded using a microplate reader.

\section{Dual-Luciferase Reporter Gene Assay}

StarBase web server v2.0 and Target scan v7.2 were used to predict the interaction among SOX2-OT, miR-942-5p and DP5. Sequence of the putative miR-942-5p target binding sequence of the wild type and mutant (Mut) 3'UTR of DP5 (Wt), and the wild type (Wt) and mutant (Mut) of SOX2-OT containing the putative binding site of miR-942-5p were synthesized and cloned into pmirGLO dual-luciferase reporter vector (Promega, Madison, WI, USA), and fused downstream of firefly luciferase gene. A total of $1 \times 10^{5}$ cells were seeded into a 12 -well plate. MiR-942-5p mimics or mock NC (100 ng) were cotransfected into HEK293 cells (Mingjin, Shanghai, China) for $6 \mathrm{~h}$ using Lipofectamine 2000 (Invitrogen, Carlsbad, CA). Luciferase activity was measured 48 $\mathrm{h}$ after transfection using the Dual-Luciferase Reporter
Assay Kit (Promega). The activity of firefly luciferase gene was normalized to the activity of Renilla luciferase gene.

\section{RNA Extraction and Quantification Real-Time PCR}

Total RNAs in cells were extracted using TRIzol reagent (Invitrogen, Carlsbad, CA). After reverse transcription reaction, qRT-PCR was performed using a ViiA ${ }^{\mathrm{TM}} 7$ realtime PCR system (Life Technologies, Grand Island, NY). GAPDH and U6 were used as internal references. The relative expression levels of each gene were calculated and normalized using the ${ }^{2-\Delta \Delta \mathrm{Ct}}$ method. Quantitative realtime PCR (qRT-PCR) was performed as described in literature. ${ }^{27}$ Primers used for qRT-PCR analysis are listed in Table 1.

\section{Western Blot}

Total proteins were extracted from transfected cells, and protein concentrations were quantified using the BCA Protein Assay Kit. Protein samples were denatured in boiling water for $10 \mathrm{~min}$ and separated using SDS-PAGE gel $(10 \%)$. After transferring to PVDF membrane, blotting was performed by incubating with anti-GAPDH $(1: 1,000$; Cell Signaling Technology, Boston, MA), DP5 (1:1,000; Cell Signaling Technology Boston, MA) at $4^{\circ} \mathrm{C}$ overnight. The anti-rabbit secondary antibody (1:5,000; Cell Signaling Technology Boston, MA) was then added and incubated for $1 \mathrm{~h}$. Western blot was carried out as described in literature. ${ }^{28}$

Table I Primers Used in This Study

\begin{tabular}{|l|l|}
\hline Name & Sequence (5' -3' $^{\prime}$ ) \\
\hline SOX2-OT & $\begin{array}{l}\text { Forward: GTAGCCTTCTGGAACGCCAATTG } \\
\text { Reverse: AGTGCAAGTGTAGGAGAATCATC }\end{array}$ \\
\hline DP5 & $\begin{array}{l}\text { Forward: ACGCATCGAGGACTTAGAGA } \\
\text { Reverse: CTGAAGATTGGCTATCGTGCC }\end{array}$ \\
\hline miR-942-5p & $\begin{array}{l}\text { Forward: GCAATGGCCTTAGCGGACAC } \\
\text { Reverse: CAGAGTCTTAGAACGAGGAC }\end{array}$ \\
\hline U6 & $\begin{array}{l}\text { Forward: AGAGAAGATTAGCATGGCCCCTG } \\
\text { Reverse: AGTGCAGGGTCCGAGGTATT }\end{array}$ \\
\hline GAPDH & $\begin{array}{l}\text { Forward: CCAAGGTCATCCATGACAAC } \\
\text { Reverse: GCTTCACCACCTTCTTGATG }\end{array}$ \\
\hline
\end{tabular}




\section{Caspase-3 Activity Analysis}

Caspase-3 activity was measured by a colorimetric assay kit (KeyGen, China). The cell protein concentration was determined by the BCA method. Samples containing equal amount of protein were incubated with $2 \mathrm{x}$ reaction buffer and specific substrate for $4 \mathrm{~h}$. The absorbance at $405 \mathrm{~nm}$ was measured on a microplate reader (HynergyTM HT, BIOTEK, Winooski, VT, USA).

\section{Annexin V-FITC/PI Double-Labeled Flow Cytometry}

Apoptosis was measured by FITC Annexin V Apoptosis Detection Kit (Vazyme, Nanjing, China). The transfected cells were collected for washing, followed by incubation with $500 \mu \mathrm{L}$ of binding buffer, $5 \mu \mathrm{L}$ of FITC Annexin $\mathrm{V}$ and $10 \mu \mathrm{L}$ of propidium iodide (PI). The apoptotic rate was determined using flow cytometry (FACS Calibur, USA). Specific experimental methods were carried out in reference to the literature. ${ }^{29}$

\section{TUNEL Analysis}

The myocardial tissues embedded in paraffin were incubated with recombinant terminal deoxynucleotidyl transferase (rTdT) solution for $1 \mathrm{~h}$. Then, it was stained using DAPI. TUNEL-stained cells were observed under a fluorescence microscope (Olympus, Fluoview 1000, Tokyo, Japan).

\section{Statistical Analyses}

Data were presented as mean \pm standard deviation (mean \pm SD). Statistical analyses were performed using the SPSS19.0 statistical software. Differences were compared with one-way ANOVA and subsequent LSD test. $P<0.05$ indicated that the difference was significant.

\section{Results}

\section{SOX2-OT Was Highly Expressed in}

\section{Doxorubicin Stimulated Primary}

\section{Cardiomyocyte}

First, whether SOX2-OT changed in heart disease and established a DOX-induced cardiomyocyte injury model was analyzed. As shown in Figure 1A and B, DOX was able to gradually decrease the cell viability of PC cells with the increasing of dose and duration of action $(P<0.01)$. As shown in Figure $1 \mathrm{C}$ and D, DOX treatment obviously promoted cell apoptosis in a dose and time-dependent manner. In addition, the expression levels of SOX2-OT were significantly increased in DOX-stimulated PC cells, and DOX treatment increased the expression levels of SOX2-OT in a dose and time-dependent manner (Figure $1 \mathrm{E}$ and $\mathrm{F})$. Considering that $\mathrm{PC}$ cells treated with $5 \mu \mathrm{M}$ DOX for $24 \mathrm{~h}$ had the most significant changes in cell viability, apoptotic rate, and the expression levels of SOX2OT, this experimental condition was chosen for subsequent experiments. These results indicated that SOX2-OT played a critical role in DOX-induced cardiomyocyte apoptosis.

\section{SOX2-OT Functioned as a Sponge of miR-942-5p in Primary Cardiomyocytes}

Starbase revealed that SOX2-OT may have a putative binding site for miR-942-5p (Figure 2A). The luciferase reporter gene assay was performed to test this hypothesis. The reported plasmid was co-transfected into HEK293 cells with miR-942-5p mimic or negative control. As shown in Fig 0.2B, overexpression of miR942-5p significantly reduced the luciferase activity of the SOX2-OT wild type vector, but it did not change the mutated vector. Furthermore, as shown in Figure 2C, the expression levels of SOX2-OT in siSOX2-OT group were significantly decreased compared with that in si-NC group $(P<0.01)$, and its expression levels in SOX2-OT group were obviously increased compared with that in NC group $(P<0.01)$. In addition, the expression levels of miR-942-5p were remarkably up-regulated in si-SOX2-OT group compared with that in si-NC group $(P<0.01)$, and its expression levels in SOX2-OT group were obviously down-regulated compared with that in NC group $(P<$ 0.01). Moreover, the expression levels of miR-942-5p were significantly decreased in the DOX-treated group in a dose and time-dependent manner (Figure 2D and E). Taken together, these results demonstrated that SOX2-OT exerted its biological function by sponging miR-942-5p.

\section{SOX2-OT Sponged and Sequestered miR-942-5p to Upregulate the Expression of DP5}

TargetScan revealed that DP5 was a potential target for miR942-5p (Figure 3A). To confirm this, the luciferase reporter gene assay was performed and found that overexpression of miR-942-5p reduced the luciferase activity of the DP5 wild type vector, but did not reduce the luciferase activity of the 

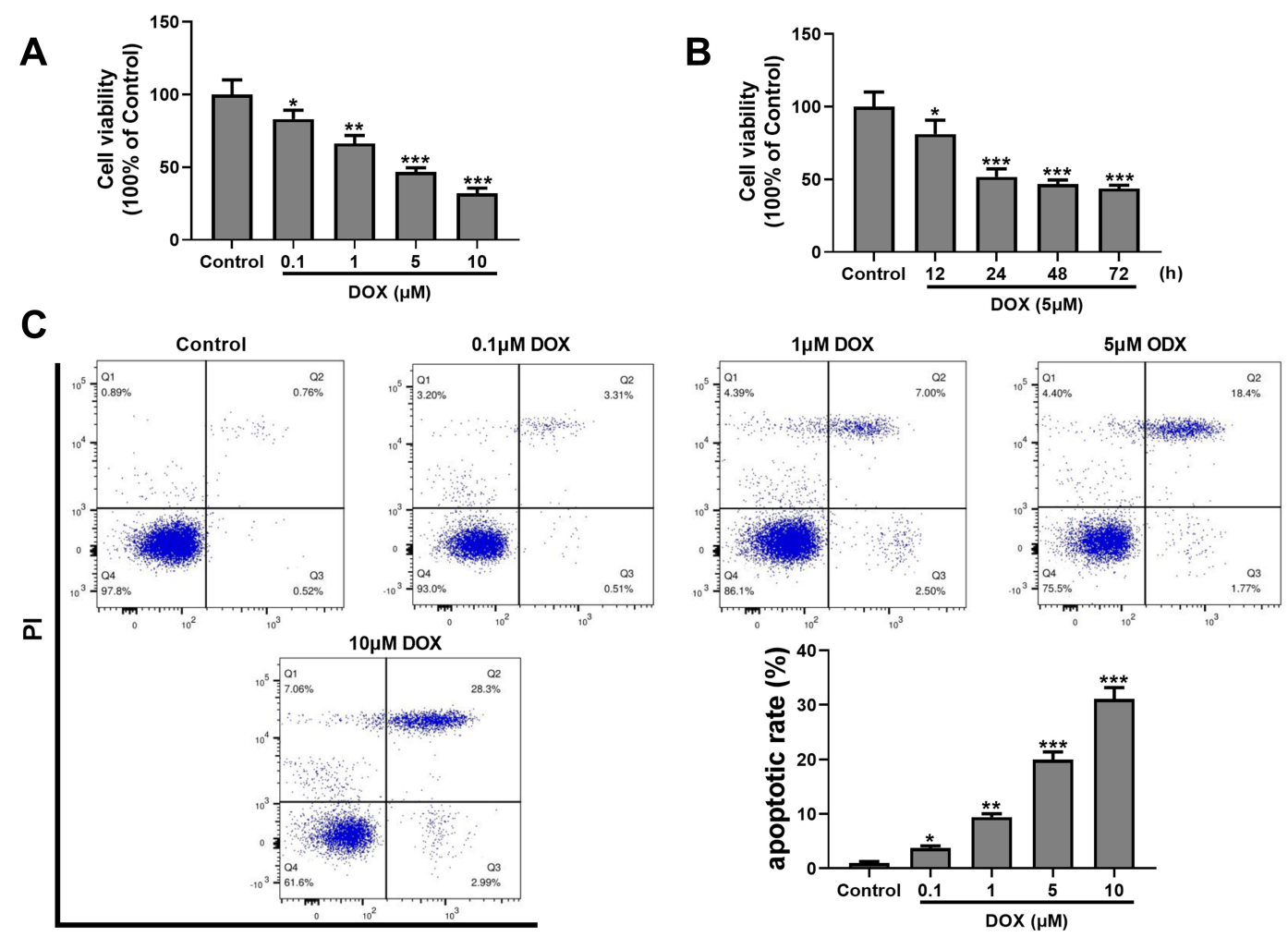

Annexin-V
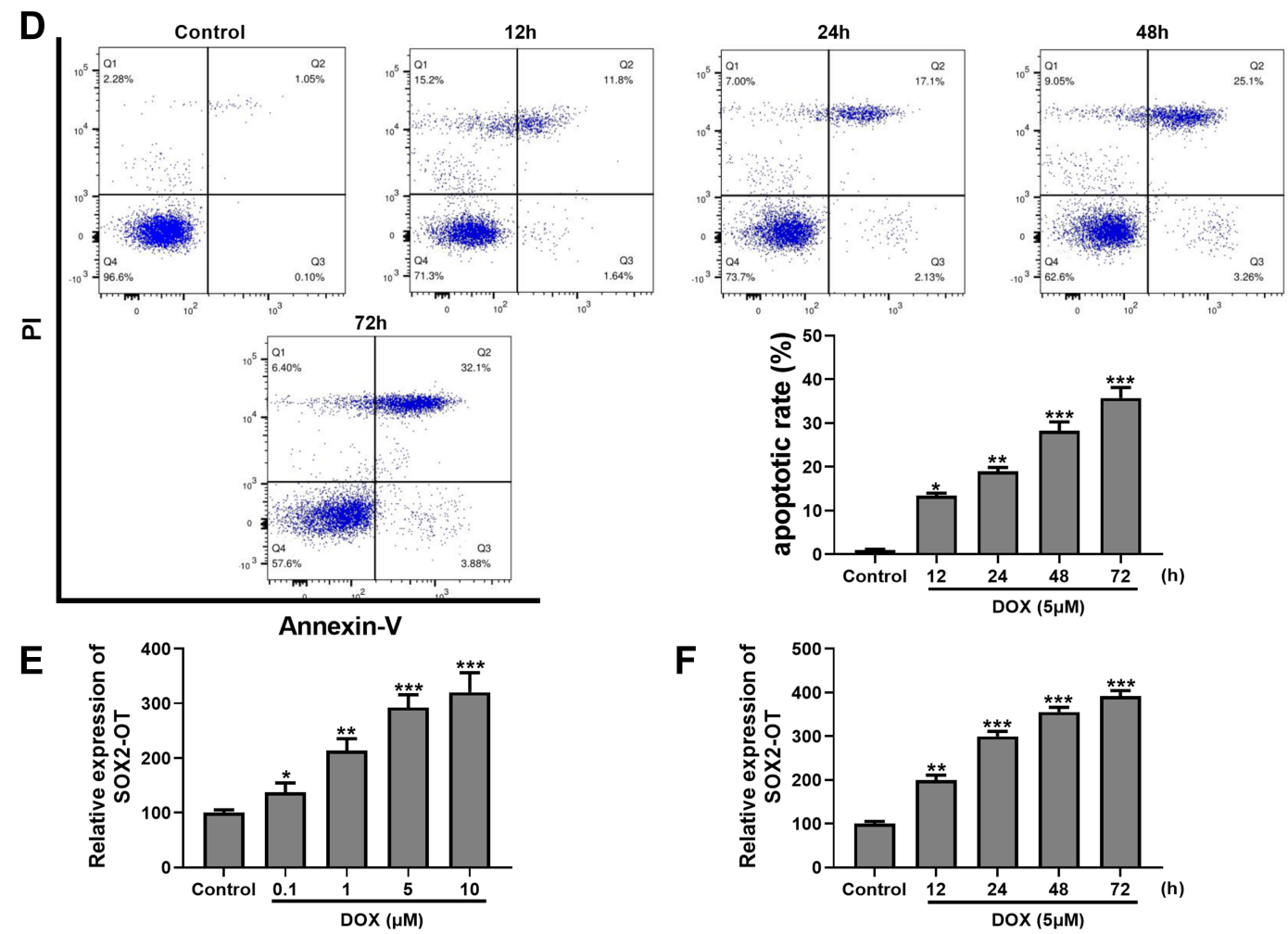

Figure I DOX treatment inhibited cell viability, promoted apoptosis, and up-regulated the expression of SOX2-OT in cardiomyocytes. (A and C and E) Cardiomyocytes were stimulated with different concentrations of DOX $(0.1,1,5,10 \mu \mathrm{M})$ for 24 h. (A) Cell viability were detected by CCK-8 assay. (C) Apoptotic cells were detected using a flow cytometry. (E) The expression of SOX2-OT was detected by RT-qPCR. (B and D and F) Cardiomyocytes were stimulated with $5 \mu$ M DOX for I2, 24, 48, and 72 h. (B) Cell viability was detected by CCK-8 assay. (D) Apoptotic cells were detected using a flow cytometry. (F) The expression of SOX2-OT was detected by RT-qPCR. *p < $0.05,{ }^{*} p<<0.01, * * * p<0.001$ vs the control group. 
A

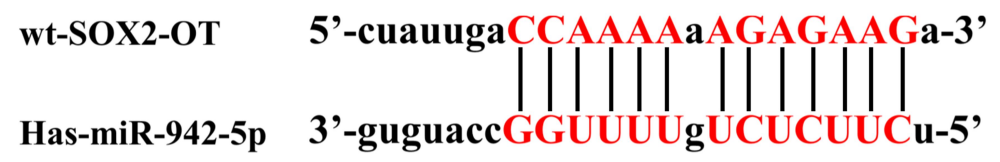

Mut-SOX2-OT 5'-cuaungaAAUUUUUUCUCUUCa-3'

C

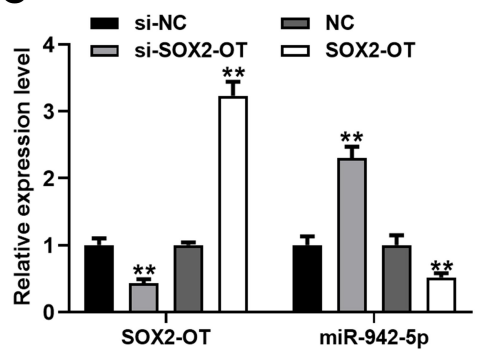

B

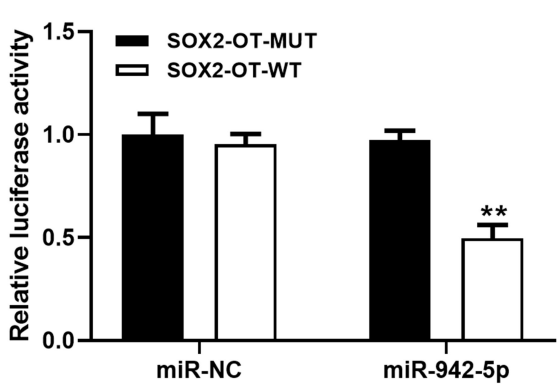

$\mathbf{E}$
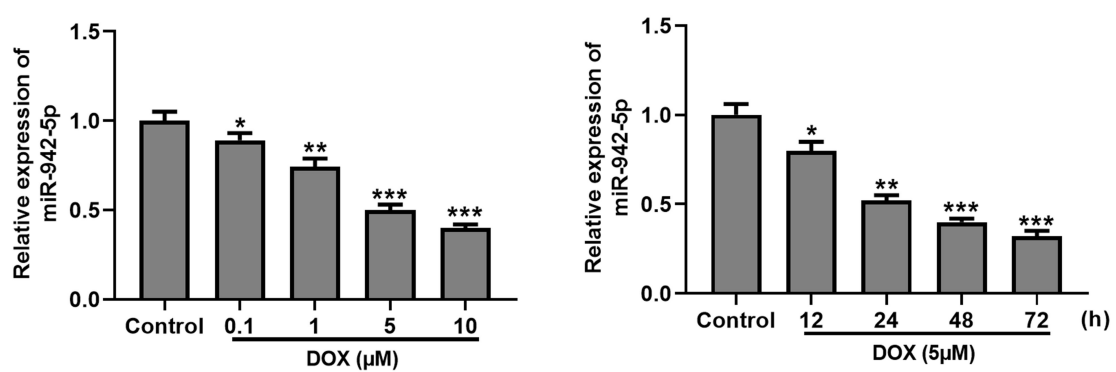

Figure 2 SOX2-OT acted as a sponge for miR-942-5p to exert biological effects. (A) Putative targeting sites for SOX2-OT and miR-942-5p. (B) Analysis of luciferase activity in HEK293 cells co-transfected with miR-942-5p mimics and SOX2-OT-WT or SOX2-OT-Mut vectors. **p < 0.01 vs SOX2-OT-MUT. (C) mRNA expression level of miR-942$5 p$ in primary cardiomyocytes knocked down or overexpressed by SOX2-OT. ** $p<0.01$ vs siNC or NC. (D) Cardiomyocytes were stimulated with different concentrations of DOX $(0.1, I, 5,10 \mu \mathrm{M})$ for $24 \mathrm{~h}$. The expression of miR-942-5p was detected by RT-qPCR. (E) Cardiomyocytes were stimulated with $5 \mu \mathrm{M}$ DOX for $12,24,48$, and 72 h. $*_{p}<0.05, *_{p}<0.01$, $* * * p<0.001$ vs the control group.

A

\author{
wt-DP5 \\ Has-miR-942-5p
}

Mut-DP5

C

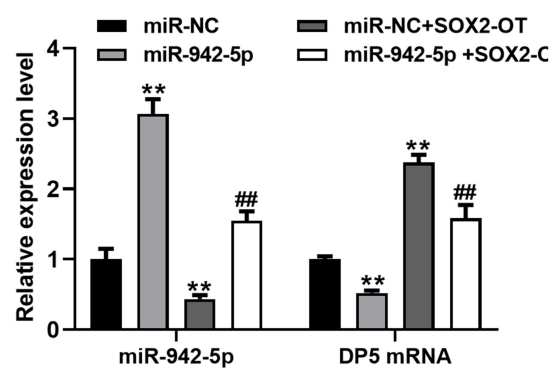

B

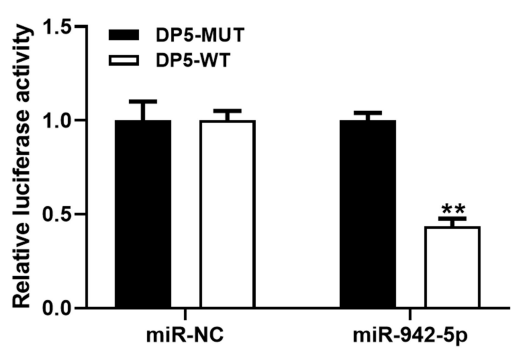

E

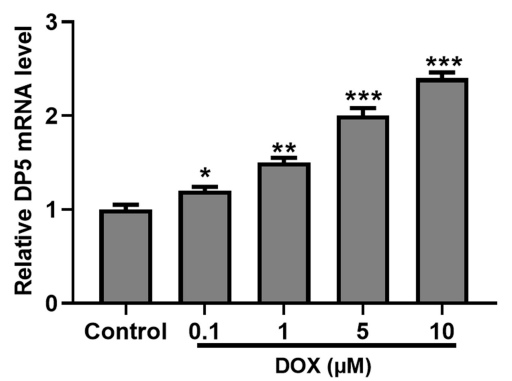

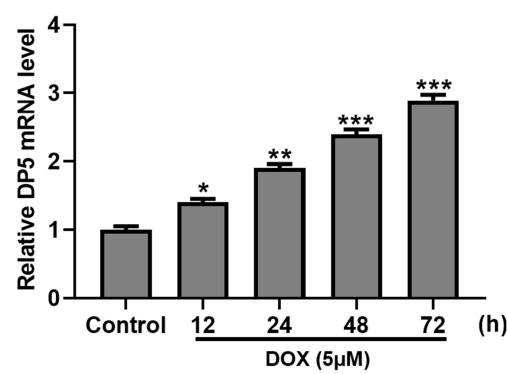

Figure 3 SOX2-OT upregulated the expression of DP5 by sponging miR-942-5p. (A) Putative binding sites for miR-942-5p and DP5 3'-UTR. (B) Analysis of luciferase activity in HEK293 cells co-transfected with miR-942-5p mimic and DP5 3'-UTR-WT or DP5 3'-UTR-Mut vector. **p < 0.01 vs DP5-WT. (C) DP5 mRNA expression levels in primary cardiomyocytes co-transfected with miR-942-5p mimic and SOX2-OT. **p < 0.01 vs miR-NC; \#p < 0.0I vs miR-NC+SOX2-OT. (D) Cardiomyocytes were stimulated with different concentrations of DOX $(0.1, \mathrm{I}, 5,10 \mu \mathrm{M})$ for $24 \mathrm{~h}$. The expression levels of DP5 were detected by RT-qPCR. (E) Cardiomyocytes were stimulated with $5 \mu$ M DOX for 12, 24, 48, and 72 h. ${ }^{*} p<0.05,{ }^{*} p<0.01$, ***p $<0.001$ vs the control group. 
mutated vector (Figure 3B). Furthermore, as shown in Figure 3C, the expression levels of miR-942-5p were significantly increased in the miR-942-5p overexpression group compared with that in the NC group $(P<0.01)$, indicating successful transfection. The expression levels of DP5 in miR-942-5p overexpression group were obviously reduced, while remarkably increased in SOX2-OT overexpression group (Figure 3C). In addition, overexpression of miR942-5p reversed the effect of overexpression of SOX2-OT on the expression of DP5 (Figure 3C). As shown in Figure 3D and E, DOX treatment significantly promoted the expression levels of DP5 in a dose and time-dependent manner. Taken together, these results demonstrated that SOX2-OT increased the expression levels of DP5 by acting as a sponge for miR-942-5p.

\section{Knockdown of SOX2-OT Inhibited Myocardial Apoptosis via the miR-942-5p/ DP5 Axis in vitro}

Next, whether SOX2-OT was involved in doxorubicininduced cardiomyocyte apoptosis was further explored. The effects of si-NC, si-SOX2-OT, miR-942-5p, or si-DP5 treatment on the apoptosis of primary cardiomyocytes were assessed. Results showed that si-NC, si-SOX2-OT, miR-942-5p, si-DP5, si-SOX2-OT + miR-942-5p, and si-SOX2-OT + si-DP5 treatment had no significant effect on cardiomyocyte apoptosis (Figure S1). As shown in Figure 4A and C, DOX significantly promoted apoptosis of primary cardiomyocytes compared with that in the control group $(P<$ 0.01). Apoptosis of primary cardiomyocytes was significantly inhibited in si-SOX2-OT group, miR-942-5p overexpression group and si-DP5 group $(P<0.01)$. Cotransfection of si-SOX2-OT with miR-942-5p reversed the effect of si-SOX2-OT on apoptosis of primary cardiomyocytes $(P<0.01)$. As shown in Figure 4B and D, DOX significantly promoted Caspase- 3 activity compared with that in the control group $(P<0.01)$. The activity of Caspase-3 was significantly inhibited in the si-SOX2-OT group, the miR-942-5p overexpression group, and the si-DP5 group $(P<0.01)$. Cotransfection of si-SOX2-OT with miR-942-5p reversed the effect of si-SOX2-OT on Caspase-3 activity $(P<0.01)$. These results indicated that si-SOX2-OT inhibited apoptosis of primary cardiomyocytes by down-regulating DP5 to up-regulate miR-942-5p.

\section{Knockdown of SOX2-OT Improved Myocardial Function Through Regulating miR-942-5p in vivo}

Four weeks after the first injection of DOX, organ samples were collected, and cardiac function was measured by echocardiography. As shown in Figure 5A and B, knockdown of SOX2-OT significantly inhibited the expression of SOX2-OT and DP5 $(P<0.01)$, while knockdown of SOX2-OT significantly increased the expression levels of miR-942-5p in cardiac tissues $(P<0.01)$. Co-transfection of si-SOX2-OT and miR-942-5p reversed the effect of siSOX2-OT on the expression of SOX2-OT, DP5 and miR942-5p in cardiac tissues $(P<0.01)$. As shown in Figure 5C and D, LVEF and LVFS scores were significantly lower after DOX treatment than that in the control group $(P<0.01)$, while LVEF and LVFS scores were significantly elevated in si-SOX2-OT group and miR942-5p overexpression group $(P<0.01)$. Co-transfection of si-SOX2-OT with miR-942-5p reversed the effect of siSOX2-OT on LVEF and LVFS scores $(P<0.01)$. TUNEL staining showed that the apoptosis rate of DOX was significantly increased compared with that in the control group $(P<0.01)$, and the apoptosis rate of miR-942-5p overexpression group was significantly decreased in siSOX2-OT group $(P<0.01)$. Co-transfection of si-SOX2OT with miR-942-5p reversed the effect of si-SOX2-OT on apoptosis rate $(P<0.01)$ (Figure 5E). Taken together, these results indicated that knockdown of SOX2-OT effectively restored cardiomyocyte apoptosis via the miR-9425p/DP5 axis.

\section{Discussion}

Doxorubicin is one of the most widely used and most effective anti-tumor drugs in clinical practice, and is often used to treat various malignant tumors and leukemias. ${ }^{30,31}$ However, the cardiotoxicity produced by the application of doxorubicin greatly limits the anti-tumor effect to a certain extent. ${ }^{32}$ With the increase of dosage, the clinical symptoms of myocardial injury gradually appear, and there is no effective prevention and treatment. Cardiotoxicity caused by doxorubicin can be divided into acute injury and chronic injury, both of which can lead to abnormal heart function, cardiomyopathy, and finally lead to severe heart failure and even death. ${ }^{33,34}$ Cardiomyocyte death caused by the accumulation of doxorubicin eventually exceeds the maximum limit that can be tolerated by normal cardiac compensation, resulting in a series of 
A

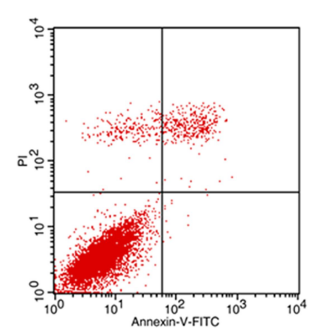

Control

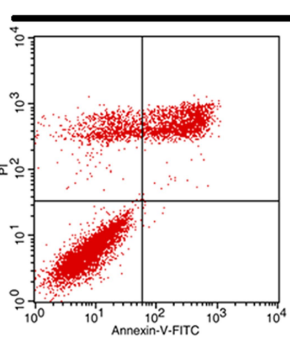

si-NC

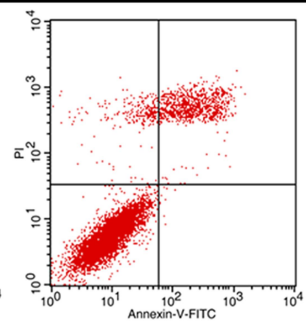

si-SOX2-OT
DOX

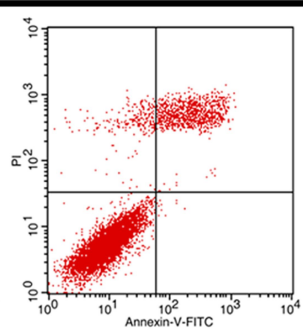

miR-942-5p

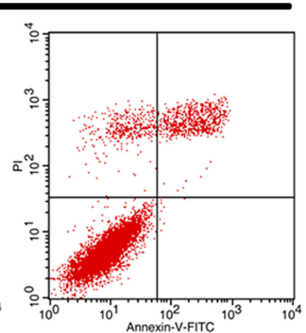

si-SOX2-OT

\section{B}
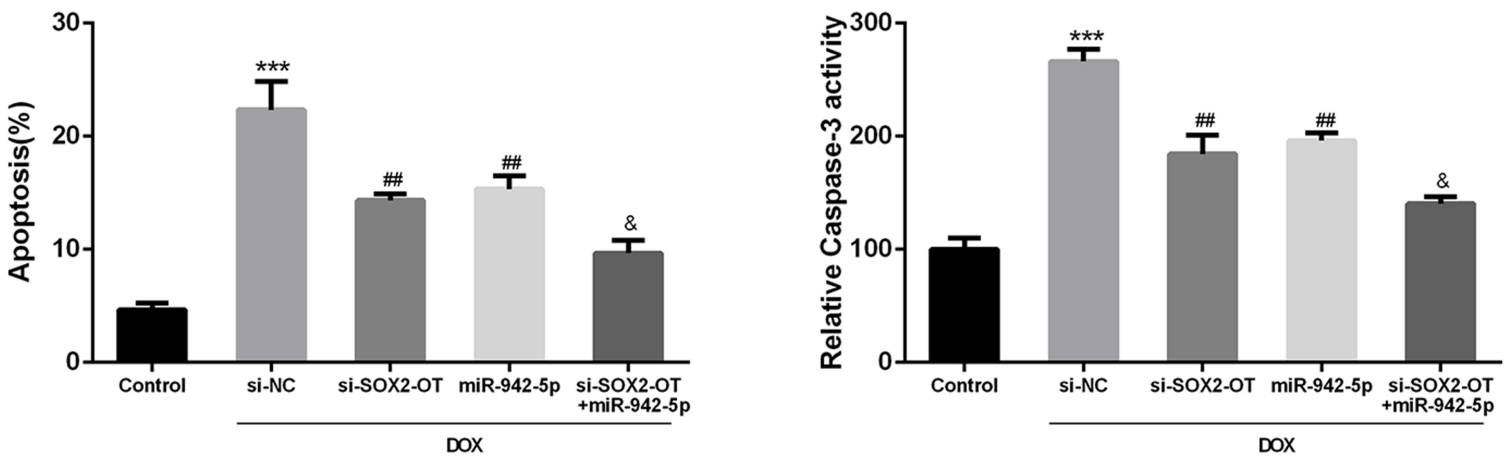

C

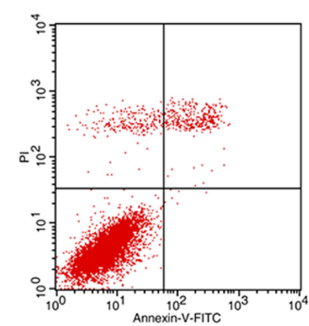

Control

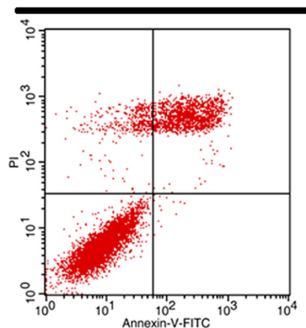

si-NC

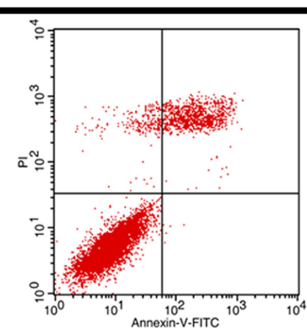

si-SOX2-OT

DOX

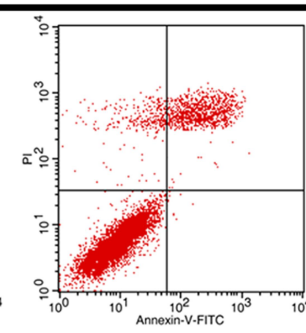

si-DP5

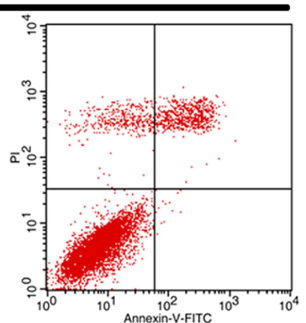

si-SOX2-OT

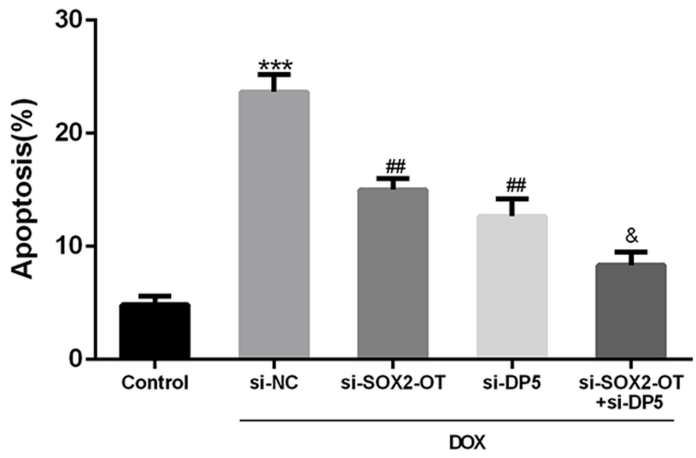

D

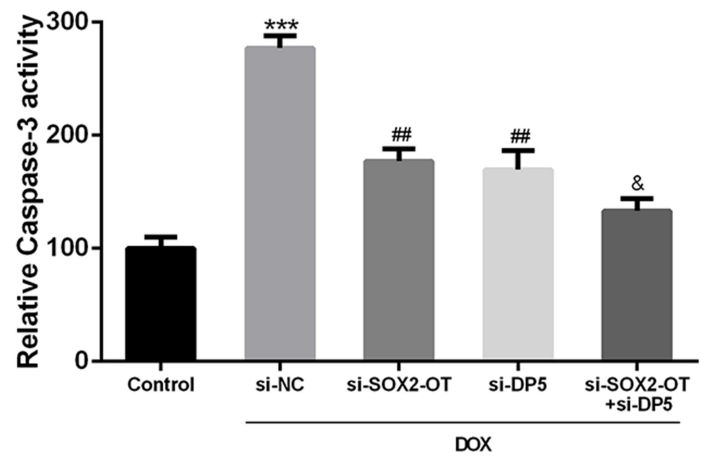

Figure 4 Biological role of SOX2-OT in DOX-induced cardiomyocyte apoptosis. Cells transfected with si-NC, si-SOX2-OT, miR-NC, miR-942-5p mimic or si-DP5 were treated with DOX for $24 \mathrm{~h}$. Apoptosis rate of primary cardiomyocytes transfected with (A and C) si-NC, si-SOX2-OT and miR-NC, miR-942-5p mimic or si-DP5. (B and D) Determination of caspase-3 activity in si-NC, si-SOX2-OT and miR-NC, miR-942-5p mimic or si-DP5 transfected primary cardiomyocytes. ${ }^{* * *} \mathrm{p}<0.00 \mathrm{I}$ vs control; ${ }^{\# \#} \mathrm{p}<0.01$ vs si-NC; ${ }^{\&}<0.05$ vs si-SOX2-OT. 
A

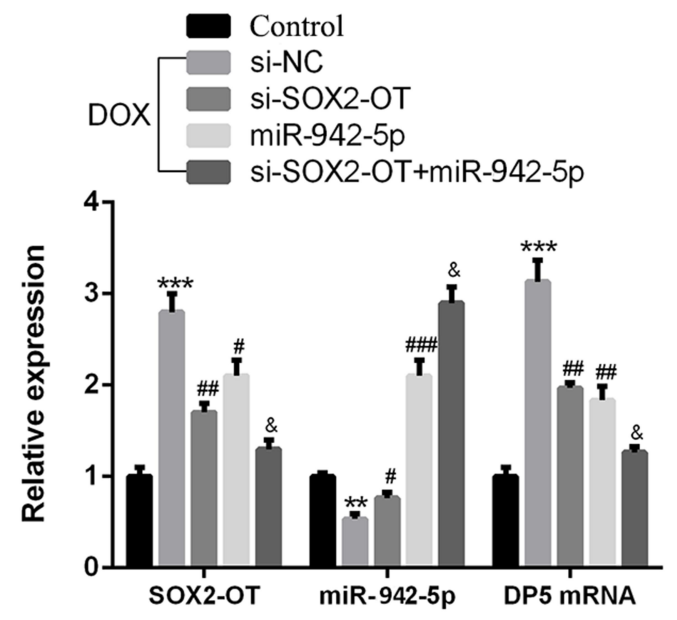

C

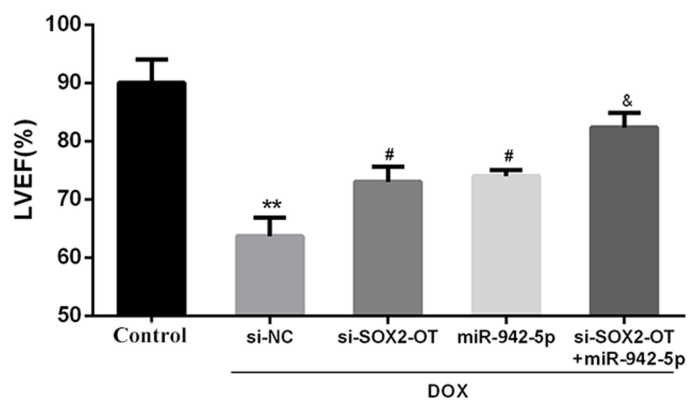

$\mathbf{E}$

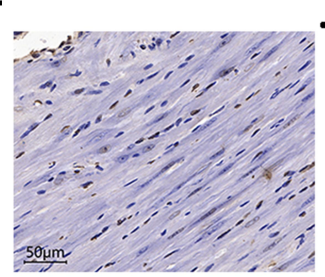

Control

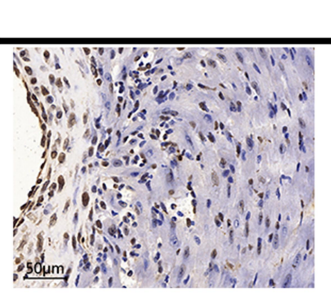

si-NC
B

DP5

GAPDH
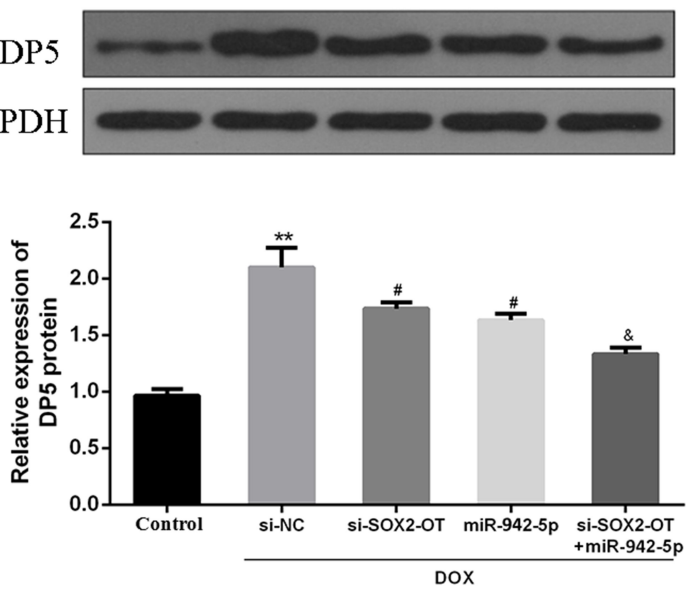

D

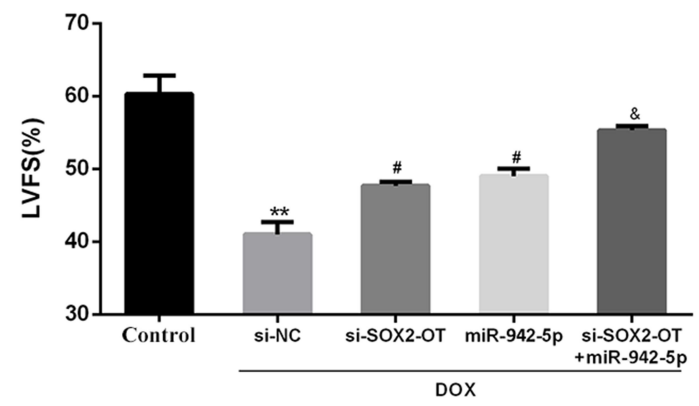

DOX

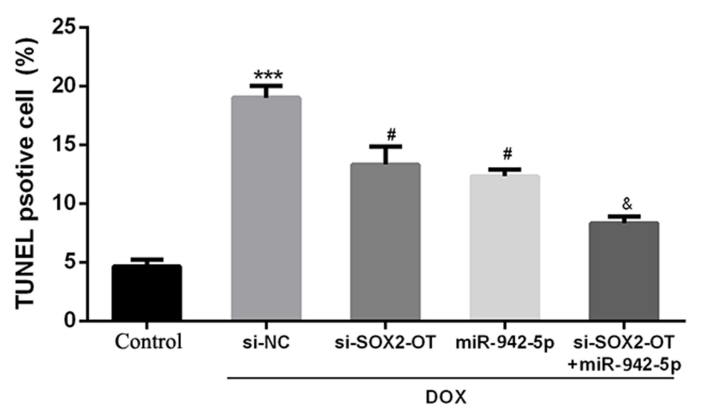

Figure 5 Effect of overexpression of SOX2-OT on myocardial function and apoptosis. (A) The expression levels of SOX2-OT, miR-942-5p and DP5. (B) Protein expression levels of DP5 in rats. Quantitative analysis of LVEF (C), and LVFS (D) in rats. (E) Apoptosis of cardiomyocytes was measured by the TUNEL method. ** $<0.0$ I and *** $p<$ 0.001 vs Control; ${ }^{\#} p<0.05,{ }^{\#} p<0.01$, and ${ }^{\# \#} p<0.00$ I vs si-NC; ${ }^{\&} p<0.05$ vs si-SOX2-OT. 
ventricular remodeling processes similar to other myocardial damage. ${ }^{35}$ Therefore, it is of great theoretical and practical value to explore new strategies for antagonizing doxorubicin-induced cardiomyocyte apoptosis at the cellular level.

Long non-coding RNAs (LncRNAs) are a class of RNA molecules. ${ }^{36}$ Studies have shown that IncRNAs can regulate gene expression and influence signaling pathways at transcriptional and post-transcriptional levels. ${ }^{37}$ Recent studies on the role of IncRNAs in the heart have been mainly focused on heart damage and remodeling, endothelial cell biology and angiogenesis. ${ }^{38}$ Studies on the role of lncRNA in cardiomyocytes found that lncRNA Bvht plays a part in the maintenance of cardiovascular developmental lineage. ${ }^{39}$ LncRNA CHRF is a newly discovered lncRNA that could serve as a target for the treatment of heart failure. $^{40}$ It has been demonstrated that IncRNA SOX2OT plays a critical role in the regulation of biological behavior of many diseases and is closely related to the prognosis of patients. ${ }^{41}$ In this study, it was found that DOX was able to inhibit the cell viability of PC cells and SOX2-OT was up-regulated after stimulation with DOX.

Studies have found that miRNAs are not only regulatory molecules for transcription and expression of genes in cells, but also signal molecules for information transmission between cells. ${ }^{42}$ In cardiovascular disease, miRNAs regulate angiogenesis, cardiomyocyte apoptosis and differentiation. MiRNAs also play a part in pathological conditions such as cardiac hypertrophy, arrhythmia, cardiac fibrosis, myocardial infarction, heart failure and cardiomyocyte apoptosis. ${ }^{20,43}$ MiR-873 was reported to suppress the translation of RIPK1/RIPK3 and inhibit RIPK1/RIPK3-mediated necrotic cell death in cardiomyocytes. MiR-873 reduces myocardial infarct size upon ischemia/reperfusion (I/R) injury in the animal model. LncRNA NRF functions as an endogenous sponge RNA and represses the expression of miR- 873 . IncRNA NRF directly binds to miR-873 and regulates the expression of RIPK1/RIPK3 and necrosis. Knockdown of NRF antagonizes necrosis in cardiomyocytes and reduces necrosis and myocardial infarction upon $\mathrm{I} / \mathrm{R}$ injury. These findings identify a novel mechanism involving NRF and miR-873 in regulating programmed necrosis in the heart and suggest a potential therapeutic avenue for cardiovascular diseases. ${ }^{44}$ MiR-942-5p has been reported to be differentially expressed in various diseases, for example, the expression levels of miR-942-5p in the serum of breast cancer patients were raised compared with that of healthy controls. ${ }^{45}$ In this study, it was found that miR-942-5p was a target gene of
SOX2OT, and knockdown of SOX2-OT increased the expression levels of miR-942-5p. But the effect of overexpression of SOX2-OT was opposite. The expression of miR942-5p was down-regulated in cells after DOX. These results indicated that SOX2-OT can exert its biological function in DOX-suppressed PC cells via spongiform miR-942-5p.

MiR-942-5p can regulate multiple apoptosis-related genes, such as ISG12a, Akt, and the NF-kB Signaling, thereby inhibiting cell proliferation and leading to apoptosis. ${ }^{46,47}$ DP5 is a newly discovered apoptosisrelated suppressor gene. The expression of DP5 is inhibited or downregulated in a variety of diseases. ${ }^{48}$ DP5 regulates the transcriptional protein AP-1, inhibits its transcriptional activity, blocks its cell-mediated signal transduction pathway, and exerts its role in inhibiting cell growth. ${ }^{49}$ This study found that DP5 was a target gene of miR-942-5p. The expression levels of DP5 were reduced with the overexpression of miR-942-5p. Cotransfection of SOX2-OT with miR-942-5p reversed the effect of overexpression of SOX2-OT on the expression of DP5. Co-transfection of si-SOX2-OT with miR-942-5p reversed the effect of si-SOX2-OT on apoptosis and Caspase-3 activity in primary cardiomyocytes. In vivo experiments showed that knockdown of SOX2-OT can inhibit the expression of SOX2-OT and DP5, while knockdown of SOX2-OT increased the expression levels of miR-942-5p in cardiac tissues. Co-transfection of siSOX2-OT with miR-942-5p reversed the effect of siSOX2-OT on the expression of SOX2-OT, DP5 and miR-942-5p in cardiac tissues. These results proved that knockdown of SOX2-OT effectively restored cardiomyocyte apoptosis via the miR-942-5p/DP5 axis.

\section{Conclusion}

Knockdown of lncRNA SOX2-OT effectively restored cardiomyocyte apoptosis through the miR-942-5p/DP5 axis. By exploring the protective effect of doxorubicin on myocardial injury, we may be able to better understand the mechanism of doxorubicin-induced cardioprotective effect. It will provide a more comprehensive theoretical basis and evidence support for clinical application as a new drug for preventing myocardial injury caused by doxorubicin.

\section{Data Sharing Statement}

The analyzed data sets generated during the study are available from the corresponding author on reasonable request. 


\section{Funding}

This study received the financial support from Natural science foundation of Guangdong Province (2015A030313441). Funder had no role in the design of the study and collection, analysis, and interpretation of data and in writing the manuscript.

\section{Disclosure}

The authors declare that they have no competing interests.

\section{References}

1. Swain SM, Whaley FS, Ewer MS. Congestive heart failure in patients treated with doxorubicin: a retrospective analysis of three trials. Cancer. 2010;97(11):2869-2879. doi:10.1002/cncr.11407

2. Lammer J, Malagari K, Vogl T, et al. Prospective randomized study of doxorubicin-eluting-bead embolization in the treatment of hepatocellular carcinoma: results of the PRECISION V study. Cardiovasc Intervent Radiol. 2010;33(1):41-52. doi:10.1007/s00270-009-9711-7

3. Lyndsay HMD, Gerald BMD, Robert BMD, et al. Liposome-encapsulated doxorubicin compared with conventional doxorubicin in a randomized multicenter trial as first-line therapy of metastatic breast carcinoma. Cancer. 2010;94(1):25-36.

4. Bartlett JJ, Trivedi PC, Pulinilkunnil T. Autophagic dysregulation in doxorubicin cardiomyopathy. $J$ Mol Cell Cardiol. 2017;104:1-8. doi:10.1016/j.yjmcc.2017.01.007

5. Kavazis AN, Morton AB, Hall SE, et al. Effects of doxorubicin on cardiac muscle subsarcolemmal and intermyofibrillar mitochondria Mitochondrion. 2017;34:9-19. doi:10.1016/j.mito.2016.10.008

6. Dokanehiifard S, Soltani BM, Ghiasi P, Baharvand H, Reza Ganjali M, Hosseinkhani S. hsa-miR-766-5p as a new regulator of mitochondrial apoptosis pathway for discriminating of cell death from cardiac differentiation. Gene. 2020;736:144448. doi:10.1016/j. gene.2020.144448

7. Akbari-Birgani S, Hosseinkhani S, Mollamohamadi S, Baharvand $\mathrm{H}$. Delay in apoptosome formation attenuates apoptosis in mouse embryonic stem cell differentiation. J Biol Chem. 2014;289 (24):16905-16913. doi:10.1074/jbc.M113.536730

8. Ghiasi P, Hosseinkhani S, Ansari H, et al. Reversible permeabilization of the mitochondrial membrane promotes human cardiomyocyte differentiation from embryonic stem cells. J Cell Physiol. 2018;234 (1):521-536. doi:10.1002/jcp. 26758

9. Mitry MA, Edwards JG. Doxorubicin induced heart failure: phenotype and molecular mechanisms. Int $J$ Cardiol Heart Vasc. 2016;10:17-24. doi:10.1016/j.ijcha.2015.11.004

10. Goyal N, Kesharwani D, Datta M. Lnc-ing non-coding RNAs with metabolism and diabetes: roles of lncRNAs. Cell Mol Life Sci. 2018;75(Suppl 1):1827-1837. doi:10.1007/s00018-018-2760-9

11. Li H, Liu X, Zhang L, et al. LncRNA BANCR facilitates vascular smooth muscle cell proliferation and migration through JNK pathway. Oncotarget. 2017;8(70):114568-114575. doi:10.18632/ oncotarget.21603

12. Wang WJ, Wang YM, Hu Y, et al. HDncRNA: a comprehensive database of non-coding RNAs associated with heart diseases. Database. 2018;2018.

13. Li Q, Zhu W, Zhang B, et al. The MALAT1 gene polymorphism and its relationship with the onset of congenital heart disease in Chinese. Biosci Rep. 2018;38(3).

14. Shafiee M, Aleyasin SA, Mowla SJ, Vasei M, Yazdanparast SA. The effect of microRNA-375 overexpression, an inhibitor of helicobacter pylori-induced carcinogenesis, on IncRNA SOX2OT. Jundishapur J Microbiol. 2016;9. doi:10.5812/jjm.23464
15. Chaudhry MA. Small nucleolar RNA host genes and long non-coding RNA responses in directly irradiated and bystander cells. Cancer Biother Radiopharm. 2014;29(3):135-141. doi:10.1089/ cbr.2013.1574

16. Zhang Y, Yang R, Lian J, Xu H. LncRNA Sox2ot overexpression serves as a poor prognostic biomarker in gastric cancer. Am J Transl Res. 2016;8(11):5035.

17. Zhong X, Coukos G, Zhang L. miRNAs in human cancer. $J$ Pathol. 2015;223(2):102-115.

18. Khraiwesh B, Zhu JK, Zhu J. Role of miRNAs and siRNAs in biotic and abiotic stress responses of plants. Biochim Biophys Acta. 2012;1819(2):137-148. doi:10.1016/j.bbagrm.2011.05.001

19. Emanueli C, Shearn AI, Angelini GD, Sahoo S. Exosomes and exosomal miRNAs in cardiovascular protection and repair. Vascul Pharmacol. 2015;71:24-30. doi:10.1016/j.vph.2015.02.008

20. Buie JNJ, Goodwin AJ, Cook JA, Halushka PV, Fan H. The role of miRNAs in cardiovascular disease risk factors. Atherosclerosis. 2016;254:S0021915016313934.

21. Cheng C, Wang Q, You W, Chen M, Xia J. MiRNAs as biomarkers of myocardial infarction: a meta-analysis. PLoS One. 2014;9(2):e88566. doi:10.1371/journal.pone.0088566

22. Goretti E, Wagner DR, Devaux Y. miRNAs as biomarkers of myocardial infarction: a step forward towards personalized medicine? Trends Mol Med. 2014;20(12):716-725. doi:10.1016/j.molmed.20 14.10.006

23. Yan Q, Shen C, Qin J, et al. HIV-1 Vpr inhibits Kaposi's sarcomaassociated herpesvirus lytic replication by inducing microRNA miR942-5p and activating NF-кB signaling. $J$ Virol. 2016;90(19):8739. doi:10.1128/JVI.00797-16

24. Cunha DA, Igoilloesteve M, Gurzov EN, et al. Death protein 5 and p53-upregulated modulator of apoptosis mediate the endoplasmic reticulum stress-mitochondrial dialog triggering lipotoxic rodent and human $\beta$-cell apoptosis. Diabetes. 2012;61(11):2763-2775. doi: $10.2337 / \mathrm{db} 12-0123$

25. Coultas L, Terzano S, Thomas T, et al. Hrk/DP5 contributes to the apoptosis of select neuronal populations but is dispensable for haematopoietic cell apoptosis. J Cell Sci. 2007;120(Pt 12):2044. doi: $10.1242 /$ jcs. 002063

26. Ishii M, Mueller I, Nakajima T, Pasquale EB, Ogawa K. EphB signaling inhibits gap junctional intercellular communication and synchronized contraction in cultured cardiomyocytes. Basic Res Cardiol. 2011;106(6):1057-1068. doi:10.1007/s00395-011-0219-3

27. Rosenwald S, Gilad S, Benjamin S, et al. Validation of a microRNA-based qRT-PCR test for accurate identification of tumor tissue origin. Mod Pathol. 2010;23(6):814. doi:10.1038/ modpathol.2010.57

28. Utermann G. Apolipoprotein E phenotyping from serum by Western blotting. Electrophoresis. 2010;7(11):492-495.

29. Eray M, Mättö M, Kaartinen M, Andersson LC, Pelkonen J. Flow cytometric analysis of apoptotic subpopulations with a combination of Annexin V-FITC, propidium iodide, and SYTO 17. Cytometry. 2015;43(2):134-142. doi:10.1002/1097-0320(20010201)43:2<134:: AID-CYTO1028>3.0.CO;2-L

30. Meng QS, Zhang PC, Yin Q, Zhang ZW, Hai-Jun YU, Ya-Ping LI. Photo-sensitive liposomes loading doxorubicin hydrochloride reverse drug resistance of breast cancer. Acta Pharmaceutica Sinica. 2017.

31. Wei Y, Gao L, Wang L, et al. Polydopamine and peptide decorated doxorubicin-loaded mesoporous silica nanoparticles as a targeted drug delivery system for bladder cancer therapy. Drug Deliv. 2017;24(1):681. doi:10.1080/10717544.2017.1309475

32. Druck M, Bar-Shlomo BZ, Gulenchyn K, et al. Radionuclide angiography and endomyocardial biopsy in the assessment of doxorubicin cardiotoxicity. Cancer. 2015;47(8):401.

33. Zhang YW, Shi J, Li YJ, Wei L. Cardiomyocyte death in doxorubicin-induced cardiotoxicity. Arch Immunol Ther Exp. 2009;57(6):435-445. doi:10.1007/s00005-009-0051-8 
34. Khiati S, Rosa ID, Sourbier C, et al. Mitochondrial topoisomerase I (Top1mt) is a novel limiting factor of doxorubicin cardiotoxicity. Clin Cancer Res. 2014;20(18):4873-4881. doi:10.1158/1078-0432. CCR-13-3373

35. Li J, Liu HP, Ramachandran S, et al. Grape seed proanthocyanidins ameliorate doxorubicin-induced cardiotoxicity. Am J Chin Med. 2010;38(03):1000806. doi:10.1142/S0192415X10008068

36. He X, Chen $X$, Zhang $X$, et al. An Lnc RNA (GAS5)/ SnoRNA-derived piRNA induces activation of TRAIL gene by site-specifically recruiting MLL/COMPASS-like complexes. Nucleic Acids Res. 2015;43(7):3712-3725. doi:10.1093/nar/gkv214

37. Guan YX, Zhang ZM, Chen XZ, Zhang Q, Liu SZ, Zhang YL. Lnc RNA SNHG20 participated in proliferation, invasion and migration of breast cancer cells via miR-495. J Cell Biochem. 2017.

38. Han P, Li W, Lin CH, et al. A long non-coding RNA protects the heart from pathological hypertrophy. Nature. 2014;514 (7520):102-106. doi:10.1038/nature13596

39. Xue Z, Hennelly S, Doyle B, et al. A G-rich motif in the lncRNA braveheart interacts with a zinc-finger transcription factor to specify the cardiovascular lineage. Mol Cell. 2016;64(1):37-50. doi:10.1016/ j.molcel.2016.08.010

40. Chen L, Yan KP, Liu XC, et al. Valsartan regulates TGF- $\beta /$ Smads and TGF- $\beta /$ p38 pathways through lncRNA CHRF to improve doxorubicin-induced heart failure. Arch Pharm Res. 2018;41(1):1-9. doi:10.1007/s12272-017-0980-4

41. Tang X, Gao Y, Yu L, et al. Correlations between IncRNA-SOX2OT polymorphism and susceptibility to breast cancer in a Chinese population. Biomark Med. 2017;11(3):277-284. doi:10.2217/bmm2016-0238
42. Noman A, Fahad S, Aqeel M, et al. miRNAs: major modulators for crop growth and development under abiotic stresses. Biotechnol Lett. 2017;39(5):685-700. doi:10.1007/s10529-017-2302-9

43. Ultimo S, Zauli G, Martelli AM, et al. Cardiovascular disease-related miRNAs expression: potential role as biomarkers and effects of training exercise. Oncotarget. 2018;9(24):17238. doi:10.18632/ oncotarget. 24428

44. Wang K, Liu F, Liu CY, et al. The long noncoding RNA NRF regulates programmed necrosis and myocardial injury during ischemia and reperfusion by targeting miR-873. Cell Death Differ. 2016;23(8):1394. doi:10.1038/cdd.2016.28

45. Zhang K, Wang YW, Wang YY, et al. Identification of microRNA biomarkers in the blood of breast cancer patients based on microRNA profiling. Gene. 2017;619:10. doi:10.1016/j.gene.2017.03.038

46. Liu N, Zuo C, Wang X, et al. miR-942 decreases TRAIL-induced apoptosis through ISG12a downregulation and is regulated by AKT. Oncotarget. 2014;5(13):4959-4971. doi:10.18632/oncotarget.2067

47. Yang D, Meng X, Xue B, Liu N, Wang X, Zhu H. MiR-942 mediates hepatitis $\mathrm{C}$ virus-induced apoptosis via regulation of ISG12a. PLoS One. 2014;9(4):e94501. doi:10.1371/journal.pone.0094501

48. Kumral A, Genc S, Ozer E, et al. Erythropoietin downregulates Bax and DP5 proapoptotic gene expression in neonatal hypoxic-ischemic brain injury. Neonatology. 2006;89(3):205-210. doi:10.1159/ 000089951

49. Chen S, Lee JM, Zeng C, Chen H, Hsu CY, Xu J. Amyloid beta peptide increases DP5 expression via activation of neutral sphingomyelinase and JNK in oligodendrocytes. J Neurochem. 2010;97 (3):631-640. doi:10.1111/j.1471-4159.2006.03774.x

\section{Publish your work in this journal}

Drug Design, Development and Therapy is an international, peerreviewed open-access journal that spans the spectrum of drug design and development through to clinical applications. Clinical outcomes, patient safety, and programs for the development and effective, safe, and sustained use of medicines are a feature of the journal, which has also been accepted for indexing on PubMed Central. The manuscript management system is completely online and includes a very quick and fair peer-review system, which is all easy to use. Visit http://www. dovepress.com/testimonials.php to read real quotes from published authors. 